\title{
Common mechanisms and holistic care in atherosclerosis and osteoporosis
}

\author{
Zoltán Szekanecz ${ }^{1 *}$, Hennie G. Raterman ${ }^{2}$, Zsófia Pethő ${ }^{1}$ and Willem F. Lems ${ }^{3}$
}

\begin{abstract}
Cardiovascular (CV) disease and osteoporosis (OP) have become increasing challenges in the aging population and even more in patients with inflammatory rheumatic diseases, such as rheumatoid arthritis, spondyloarthropathies, and systemic lupus erythematosus. In this review, we discuss how the epidemiology and pathogenesis of CV events and OP are overlapping. Smoking, diabetes mellitus, physical inactivity as conventional risk factors as well as systemic inflammation are among the modifiable risk factors for both CV events and bone loss. In rheumatic patients, systemic "high-grade" inflammation may be the primary driver of accelerated atherogenesis and bone resorption. In the general population, in which some individuals might have low-grade systemic inflammation, a holistic approach to drug treatment and lifestyle modifications may have beneficial effects on the bone as well as the vasculature. In rheumatic patients with accelerated inflammatory atherosclerosis and bone loss, the rapid and effective suppression of inflammation in a treat-to-target regime, aiming at clinical remission, is necessary to effectively control comorbidities.
\end{abstract}

Keywords: Atherosclerosis, Osteoporosis, Bone loss, Inflammation, Risk factors, DXA, Rheumatoid arthritis

\section{Introduction}

Multimorbidity may become an increasing challenge in society [1]. In a study of more than 1.7 million individuals in Scotland, $42 \%$ had one or more morbidities and $23 \%$ were multimorbid [2].

In the last decades, both cardiovascular $(\mathrm{CV})$ disease (CVD) and osteoporosis (OP) have been acknowledged as crucial health problems. Both diseases have a major impact on daily clinical functioning and quality of life and most importantly on life expectancy compared with the general population. Thus, CVD and OP should be considered major health issues [1-3].

CVD and OP may occur simultaneously in the general population under non-inflammatory conditions. Both CVD and OP have also been associated with autoimmune and inflammatory rheumatic diseases, such as rheumatoid arthritis (RA), spondyloarthropathies (SpAs), or systemic lupus erythematosus (SLE) [1, 4-15]. Immuno-inflammatory processes may accelerate atherosclerosis and bone loss under non-inflammatory conditions as well as in inflammatory

\footnotetext{
* Correspondence: szekanecz.zoltan@med.unideb.hu

${ }^{1}$ Division of Rheumatology, Department of Internal Medicine, Faculty of Medicine, University of Debrecen, Nagyerdei street 98, Debrecen 4032, Hungary

Full list of author information is available at the end of the article
}

rheumatic diseases $[4,16-18]$. Immunosuppression including targeted therapies aiming at clinical remission may improve CVD and OP secondary to rheumatic diseases [4-6, 19].

In this review, we will first briefly discuss the common pathogenic mechanisms in atherosclerosis and bone loss. As numerous cells and mediators are implicated in these processes, we will give only a general overview of these processes. Then we will discuss the two sides of the story: clinical and epidemiological evidence of increased $\mathrm{CV}$ risk in OP and bone loss and fragility fractures (FFs) in patients with CVD. As both CVD and OP are highly accelerated by systemic inflammation and autoimmunity, we will briefly present the "Bermuda triangle" of CVD, OP, and inflammatory rheumatic diseases (Fig. 1). We will choose RA as a prototype as the greatest amount of information is available on this disease. Finally, we will present some evidence that atherosclerosis, OP, and-if present-inflammation may be simultaneously and effectively targeted. The aim of this review is to describe the most relevant common mechanisms between bone loss and atherosclerosis under both non-inflammatory and inflammatory conditions. We also wish to put forward the idea of a holistic approach during disease

(C) The Author(s). 2019 Open Access This article is distributed under the terms of the Creative Commons Attribution 4.0 International License (http://creativecommons.org/licenses/by/4.0/), which permits unrestricted use, distribution, and 


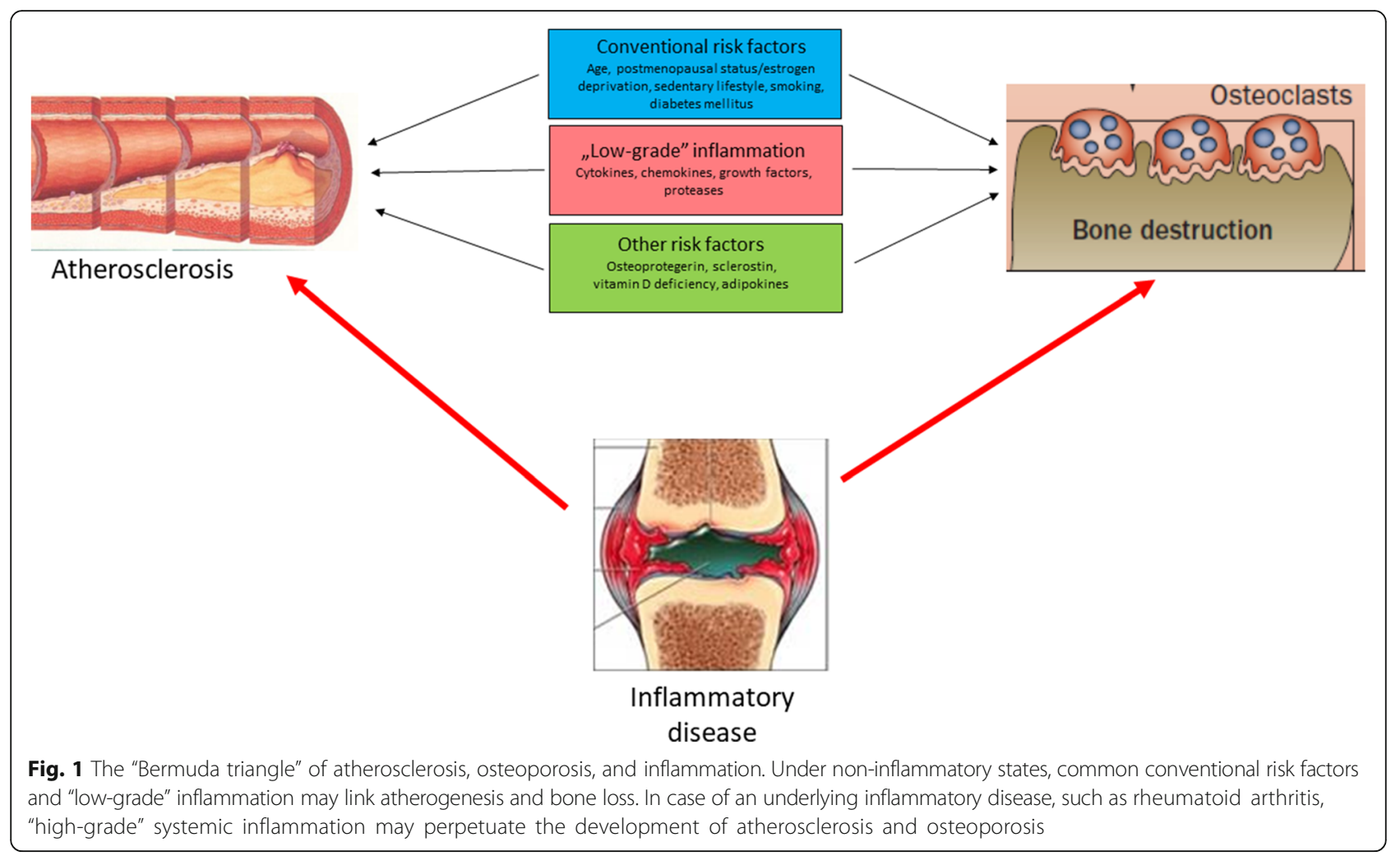

prevention and therapy. We want to present a comprehensive review that discusses most aspects of this topic. Therefore, owing to space limitations, not all issues will be discussed in great detail.

\section{Common mechanisms in the development of atherosclerosis and bone loss Conventional risk factors}

Atherosclerosis/CVD and OP may share numerous risk factors (Table 1). Some conventional risk factors are relevant for both OP FF and CV events. Aging is by far the most important, but postmenopausal status or estrogen deprivation (or both), physical inactivity, and smoking also play major roles $[20,21]$.

In addition, diabetes mellitus (DM) not only is a well-known risk factor for CVD [22] but also has a negative effect on bone strength and increased incidence of FF, as has been shown recently [23]. There is evidence that type $1 \mathrm{DM}$ (T1DM) is associated with lower bone mass, lack of insulin and insulin-like growth factor-1, and increased levels of pro-inflammatory cytokines leading to FF. In T2DM, in spite of increased bone mass, bone strength is decreased because of decreased bone formation, accumulation of advanced glycation end products (AGE), and cortical porosity. The paradox of higher bone mineral density (BMD) with increased FF risk in T2DM may also be explained by more frequent falls due to, among other things, diabetic retinopathy and polyneuropathy in addition to the poorer bone quality [23].

Additional conventional $\mathrm{CV}$ risk factors also associated with increased risk of low BMD may include dyslipidemia and hypertension [20]. However, the current evidence supporting the role of these factors in linking CVD and OP is not conclusive [20].

\section{Inflammatory risk factors}

In addition to traditional risk factors, systemic inflammation has been implicated in both atherosclerosis and bone loss. Inflammatory cells, acute phase reactants (for example, C-reactive protein, CRP; erythrocyte sedimentation rate, ESR), several pro-inflammatory cytokines, chemokines, and growth factors and other mediators-including osteoprotegerin (OPG), receptor activator of nuclear factor kappa B ligand (RANKL), and sclerostin (SOST) - are associated with both events [5, 16-18, 21, 24, 25].

"Low-grade inflammation"-including immune cells ( $\mathrm{T}$ and $\mathrm{B}$ cells, macrophages, and endothelial cells) and inflammatory mediators-plays an important role in the pathogenesis of both atherosclerosis and OP. Furthermore, inflammatory rheumatic diseases, such as RA, SpA, or SLE, have been associated with secondary atherosclerosis, increased $\mathrm{CV}$ morbidity and mortality, and increased bone loss $[4,5,7,8,18,19,21]$. As described later, in such "high-grade inflammatory" states, both atherosclerosis and bone loss are even further accelerated, 
Table 1 The most imprtant risk factors for atherosclerosis and osteoporosis ${ }^{*}$

\begin{tabular}{|c|c|c|}
\hline & Atherosclerosis & Osteoporosis \\
\hline Age & $\begin{array}{l}+++ \\
{[21,24]}\end{array}$ & $\begin{array}{l}+++ \\
{[21,24]}\end{array}$ \\
\hline Dyslipidemia & $\begin{array}{l}+ \\
{[20]}\end{array}$ & $\begin{array}{l} \pm \\
{[20]}\end{array}$ \\
\hline Hypertension & $\begin{array}{l}+ \\
{[20]}\end{array}$ & $\begin{array}{l} \pm \\
{[20]}\end{array}$ \\
\hline Smoking & $\begin{array}{l}+ \\
{[21,24]}\end{array}$ & $\begin{array}{l}+ \\
{[21,24]}\end{array}$ \\
\hline Diabetes mellitus & $\begin{array}{l}+ \\
{[22]}\end{array}$ & $\begin{array}{l}+ \\
{[22]}\end{array}$ \\
\hline Sedentary lifestyle/immobility & $\begin{array}{l}+ \\
{[21,24]}\end{array}$ & $\begin{array}{l}+ \\
{[21,24]}\end{array}$ \\
\hline Vitamin $D$ deficiency & $\begin{array}{l} \pm \\
{[42]}\end{array}$ & $\begin{array}{l}+ \\
{[42]}\end{array}$ \\
\hline Postmenopausal status/estrogen deprivation/premature menopause & $\begin{array}{l}+ \\
{[21,24]}\end{array}$ & $\begin{array}{l}+ \\
{[21,24]}\end{array}$ \\
\hline "Low-grade inflammation" & $\begin{array}{l}++ \\
{[4,5,17,18]}\end{array}$ & $\begin{array}{l}++ \\
{[6,16]}\end{array}$ \\
\hline Falls & - & $\begin{array}{l}+ \\
{[27]}\end{array}$ \\
\hline Inadequate calcium intake & - & $\begin{array}{l}+ \\
{[21]}\end{array}$ \\
\hline Alcohol consumption & - & $\begin{array}{l}+ \\
{[21]}\end{array}$ \\
\hline Hypertension & $\begin{array}{l}+ \\
{[21]}\end{array}$ & - \\
\hline Stress & $\begin{array}{l}+ \\
{[21]}\end{array}$ & - \\
\hline
\end{tabular}

${ }^{*}$ References in [ ]

and treatment of the underlying inflammatory disease, primarily by targeted therapy, may have beneficial effects on the bone and vasculature $[4,6,19,26]$. In general, among inflammatory mediators, CRP, pro-inflammatory cytokines (for example, tumor necrosis factor alpha [TNF- $\alpha]$ and interleukin 1 (IL-1), IL-6, IL-17, and IL-12/IL-23), some chemokines, growth factors, adipokines, and the renin-angiotensin-aldosterone system are important factors of atherogenesis and bone resorption [5, 6, 17, 18, 21, 24, 27-29]. For example, elevated CRP, TNF- $\alpha$, and IL- 6 levels have been associated with both myocardial infarction (MI) and non-traumatic FF $[21,29,30]$.

Adipokines-primarily adiponectin, leptin, resistin, and chemerin-have been implicated in atherosclerosis under both inflammatory and non-inflammatory conditions [31, 32]. Various adipokines may also be involved in bone remodeling [31,33]. For example, Varri et al. [34] performed a study on 290 postmenopausal women in Finland, in which adiponectin levels were inversely correlated with total body and femoral neck BMD but showed no relationship with coronary artery calcification $(\mathrm{CAC})$ or carotid artery intima-media thickness
(cIMT). In addition, leptin levels were associated with CAC [34].

The activated renin-angiotensin system (RAS) promotes atherogenesis. Angiotensin II also activates osteoclasts, while angiotensin-converting enzyme inhibitors may increase BMD and reduce FF risk [24, 35].

\section{Factors primarily involved in bone metabolism}

OPG and SOST are known factors related to bone biology. However, they have also been associated with $\mathrm{CV}$ events [36, 37]. OPG is a decoy receptor for RANKL that inhibits osteoclast activation and bone resorption [37]. In addition, OPG has been implicated in vascular calcification, matrix rearrangement, diabetic retinopathy, and most importantly in atherosclerosis, plaque destabilization, and CVD [36]. RANKL is overexpressed in vulnerable atherosclerotic plaques and may be able to reduce the risk of plaque rupture [24]. OPG has been related to CV morbidity and mortality [24, 38].

SOST is a known inhibitor of Wnt-mediated osteoblast activation and bone formation. Thus, SOST is involved in bone resorption and inflammatory bone loss [37, 39]. SOST has been associated with CV mortality in patients 
on hemodialysis [21]. Recently, SOST has been identified as a possible marker for early atherosclerosis [40].

Bone morphogenetic proteins (BMPs) induce mesenchymal cell differentiation toward the osteoblastic lineage. Various BMPs, primarily BMP-2 and BMP-7, stimulate collagen synthesis and are involved in bone formation. BMPs have been implicated in atherosclerosis. BMP-2 is expressed by vascular endothelial and smooth muscle cells. BMP expression is upregulated in the atherosclerotic plaques. Moreover, vascular BMP-2 expression is regulated by pro-inflammatory stimuli, such as TNF- $\alpha$. BMP- 4 has been associated with atherosclerosis and hypertension [24, 41].

Vitamin D may be considered both traditional and immuno-inflammatory risk factors for atherosclerosis with OP. Vitamin D deficiency has been implicated in bone loss and also in atherosclerosis [42, 43]. With regard to CVD, vitamin D deficiency may indirectly promote atherosclerosis on the basis of its relationship with DM, dyslipidemia, and hypertension. However, the exact mechanisms through which low vitamin D levels may directly lead from endothelial dysfunction to MI or stroke are not yet fully understood. Recent studies suggested that pathways potentially targeted by vitamin $\mathrm{D}$ include vascular muscle contractility, inflammatory mechanisms, oxidative stress, and the uptake of cholesterol by macrophages $[44,45]$.

Parathyroid hormone (PTH) is involved in age-related bone loss. PTH and PTH-related protein may also exert $\mathrm{CV}$ effects. Primary hyperparathyroidism has been associated with hypertension as well as increased CV morbidity and mortality $[21,24]$.

Fibroblast growth factor 23 (FGF-23) regulates phosphorous metabolism. Phosphorous may exert direct toxic effects on the vasculature. FGF-23 and one of its receptors, klotho, have been implicated in vascular calcification in patients on hemodialysis (see later). In addition, klotho deficiency and increased FGF-23 may lead to transformation of endothelial cells into osteoblast-like cells and mineralization. In contrast, high klotho expression inhibits vascular calcification [21, 46]. Even in individuals with normal renal function, high FGF-23 and low klotho levels may be associated with increased CV morbidity and mortality $[21,46]$ as well as OP and FF [21, 47].

Osteopontin (OPN) is an extracellular structural protein stimulated by $1,25-\mathrm{OH}$-vitamin $\mathrm{D}_{3}$. OPN promotes bone resorption via several molecular mechanisms. OPN is an inhibitor of ectopic calcification and vascular calcification $[24,48]$.

Cathepsin $\mathrm{K}$ exerts an important role in osteoclast activation and extracellular matrix degradation. Cathepsin $\mathrm{K}$ stimulates the degradation of type I and II collagens and osteonectin. It induces bone loss. Moreover, disruption of cathepsin $\mathrm{K}$ reduces plaque formation and the progression of atherosclerosis [24, 49].

\section{Vascular calcification}

Vascular medial wall calcification has been associated mainly with chronic kidney disease (CKD) [50]. The process involves impaired calcium and phosphate homeostasis, dysregulated PTH production, and abnormal calcium sensing $[50,51]$. Recent studies have evaluated the role of Runx2, RANKL, OPG, and FGF-23 in CKD-associated vascular calcification [50, 51]. For example, as discussed above, high serum FGF-23, high phosphorous, and low klotho levels have been associated with increased arterial calcification in patients on hemodialysis [21]. Arterial calcification in CKD has been associated with low BMD [52] as well as impaired trabecular bone microarchitecture [53].

\section{Surrogate markers indicating a relationship between atherosclerosis and bone loss}

Certainly, the major issue is whether there is epidemiological and clinical evidence for increased $\mathrm{CV}$ risk in $\mathrm{OP}$ and increased risk of OP and FF in patients with CVD. Studies with significant clinical endpoints (MI, stroke, FF, and even mortality) are necessary to answer these two questions. It is important to note that in addition to clinical association studies described later, preliminary investigations using surrogate markers of subclinical CVD (for example, cIMT and CAC) were conducted in order to demonstrate associations with BMD. For example, Barengolts et al. [54] assessed coronary calcium burden by electron-beam computed tomography (CAC) and lumbar spine BMD in 45 postmenopausal women. Higher calcium scores were associated with lower BMD [54]. Campos-Obando et al. [55] assessed BMD and CAC during a mean of 6.4 years of follow-up in the Rotterdam Study. Increased BMD loss was associated with higher CAC scores in women but not in men. On the other hand, no associations between $\mathrm{CAC}$ and fracture risk could be observed [55]. Shaffer et al. [56] correlated cIMT and BMD in 535 women and 335 men. In the subset of individuals older than 60 years, BMD inversely correlated with cIMT [56]. Finally, Delgado-Frias et al. [57] found an inverse correlation between OPG levels and brachial artery flow-mediated vasodilation (FMD), a marker of endothelial function in patients with RA. These studies using surrogate markers already suggest that atherosclerosis/CVD and bone loss/BMD may be associated.

In conclusion, numerous non-inflammatory (traditional) and inflammatory factors may link atherosclerosis and osteoporosis. Some of these factors may be used as surrogate markers of the two interrelated processes. 


\section{Increased cardiovascular risk in osteoporosis}

As mentioned in the previous section, OP and CVD have common pathophysiological links and risk factors $[21,24]$. Epidemiological evidence has been found between an increased risk and prevalence of CVD in patients with OP [24]. In the recent meta-analysis (of 25 studies with 10,300 patients) performed by Ye et al. [58], the adjusted incidence of any atherosclerotic vascular abnormality was significantly increased in individuals with low BMD versus normal BMD (odds ratio (OR) 2.96) as well as in those with OP versus without OP (OR 2.45) [58]. Veronese et al. [59] performed a meta-analysis of 11 studies indicating that people with low BMD are at increased risk of developing CVD during follow-up with an adjusted hazard ratio (HR) of 1.33 [59]. Moreover, this meta-analysis observed that each decrease of BMD by one standard deviation (SD) increased the CVD risk by $16 \%$ (HR 1.16). In regard to specific CVD types, each BMD decrease by one SD corresponded to a consecutively increased risk of $\mathrm{CV}$ and cerebrovascular disease by $44 \%$ and $28 \%$, respectively [59]. In contrast, Jin et al. [60] published data from the recent Korean STROBE (Strengthening the Reporting of Observational studies in Epidemiology) study. They could not completely confirm the observed association between cerebrovascular disease and OP, as vertebral or hip BMD was not associated with cerebral arterial disease [60].

With respect to associations between FF and CVD, Veronese et al. [59] reported a significant association between FF at baseline and subsequent development of CVD (HR 1.20). FF at baseline was also associated with an increased risk of cerebrovascular conditions and death due to CVD [59]. In contrast, in a much earlier study, Varosy et al. [61] unexpectedly found a decreased risk of subsequent coronary events in 2700 postmenopausal women with known CVD and skeletal fractures. In this study, the risk of developing further coronary events during 4 years' follow-up was reduced by $25 \%$ in women with FF compared with those without fractures (HR 0.74). It is important to note that these patients already had prevalent CVD in addition to FF at baseline [61]. Therefore, the exact association between FF and later CVD needs to be determined by conducting larger prospective studies [62].

Some studies also assessed CV mortality in patients with OP. In the meta-analysis by Qu et al. [63], an increased risk of $\mathrm{CV}$ mortality was described in patients with OP. A significant relationship between low BMD at all sites and CV mortality was found. On the other hand, this study could not demonstrate an association between cerebrovascular mortality and OP [63]. In a prospective study of more than 700 individuals, Domiciano et al. [64] observed increased CVD mortality in patients with
OP as defined by total hip T-score (HR 3.17). Moreover, this study suggested that a one-SD decrease of total hip BMD was a predictor for all-cause mortality in elderly people [64]. These findings are in line with some early studies that reported increased CV mortality in patients with OP $[21,24]$. However, the recent meta-analysis by Veronese et al. [59] suggests that publication bias may have influenced some of the abovementioned results, so the precise $\mathrm{HR}$ of OP on CVD mortality remains to be elucidated [59].

In conclusion, even in non-inflammatory states and despite some controversial reports, there is increased risk of atherosclerosis and CVD in individuals with OP. Several pathogenic processes may link atherosclerosis to OP. Moreover, patients with OP should be routinely screened for atherosclerosis and CVD.

\section{Increased risk of osteoporosis and fragility fractures in cardiovascular disease}

There is evidence suggesting that patients with CVD have an increased risk of bone loss and FF. Den Uyl et al. [65] performed a systematic review of seven population cohort studies on patients with subclinical CVD. Six of the highest-ranked studies that included a mean of 2000 patients indicated that subjects with prevalent subclinical CVD disease had higher risk for increased bone loss and FF compared with individuals without CVD. This was observed in both women and men. Both vertebral and hip FFs were observed. The overall HR/OR was 2.3 to 3.0. The data also suggested that subjects with low BMD had higher $\mathrm{CV}$ mortality rates and incident $\mathrm{CV}$ events than those with normal BMD. In this analysis, the most important risk factors were age, estrogen deficiency, and inflammation [65].

Sennerby et al. [66] followed almost 32,000 Swedish twins from the age of 50 years for a mean follow-up time of 20 years. None of the subjects had CVD at this age. The main outcome measure was time to hip fracture after diagnosis of CVD. The crude absolute rates of FF per 1000 person-years after the diagnosis of heart failure, stroke, peripheral arterial disease, and ischemic heart disease compared with no-CVD were 12.6, 12.6, 6.6, and 5.2 versus 1.2 , respectively. The adjusted HRs of hip FF in these CVDs were 4.4, 5.1,3.2, and 2.3, respectively. Thus, CVD was significantly associated with subsequent FF [66].

Gerber et al. [67] followed 3321 patients with incident MI and 3321 controls until an FF or death occurred. The overall FF HR in MI patients was 1.32. This HR increased according to time period: in 1979-1989, 19901999, and 2000-2006, the HRs were 0.81, 1.47, and 1.73, respectively [67]. These changes could be explained by increasing age over time as well as other environmental factors. 
Finally, Pouwels et al. [68] assessed the risk of hip/ femur FF after stroke. The adjusted ORs of FF in any stroke and hemorrhagic, ischemic or undefined stroke were $1.96,1.94,1.85$, and 2.10 , respectively, compared with those subjects who never experienced stroke [68].

In conclusion, there is increased risk of bone loss, OP, and FF in patients with CVD. A number of mechanisms may lead to bone loss in CVD. Furthermore, patients with CVD should be regularly screened for OP.

\section{Cardiovascular disease and osteoporosis in inflammatory rheumatic diseases}

Patients with inflammatory rheumatic diseases not only may suffer from their disease but also may have comorbidities [1, 13]. The greatest amount of information has become available in RA [1, 4, 13]; therefore, we will mostly discuss this disease with implications for other inflammatory rheumatic diseases. For example, in the large COMORA (Comorbidities in Rheumatoid Arthritis) study of 4586 patients with RA in 17 countries, a high prevalence of comorbidities and their risk factors was found, but the variability between countries in the prevalence and also in the detection of comorbidities was highly variable [13]. In a recent prospective Swedish cohort of 950 patients with early RA, factors associated with the development of one or more comorbidities, including CVD, stroke, and OP, were analyzed. Disease activity and ESR were among the most common risk factors for comorbidities, indicating the importance of inflammation [25].

As already mentioned above, both CVD and OP have been associated with RA as well as other types of arthritis (for example, $\mathrm{SpA}$ ) and autoimmune connective tissue diseases (for example, SLE) [1, 4-15, 69]. The risks of both CVD and FF are increased, roughly doubled in RA, carrying a large impact on the quality of life in these patients $[4,8,13,15,69]$. Again, the crucial question is whether these comorbidities, as described above, are also interrelated under inflammatory conditions. Relatively few studies have been carried out in RA or SLE assessing whether a fracture would be a risk factor for a CV event or CVD would increase OP or FF risk.

With respect to CVD and OP in arthritis and autoimmune patients, a recent population-based cohort study by Ni Mhuircheartaigh et al. [70] indicated a substantial increase of CVD development in RA patients with FF. In this study, 1171 incident RA patients compared with 1171 non-RA subjects with a sustained FF were followed for 12 years for further CVD development. In controls, FF was not associated with increased $\mathrm{CV}$ risk (HR 1.10-1.12). However, in RA subjects with FF, significantly increased $\mathrm{CV}$ risk was shown (HR 1.81 for FF and 1.80 for major OP fracture subjects) [70]. To the best of our knowledge, no other studies on FF and CVD development in rheumatic conditions have been performed.

With respect to non-FF studies, Popescu et al. [71] reported that patients with RA and CV comorbidity had lower total bone mass. Provan et al. [72] presented 15-year follow-up data from the Oslo RA Register. RA patients, who deceased from primary atherosclerotic death including both $\mathrm{CV}$ or cerebrovascular disease, were more often suffering from OP (57\% versus 36\%) and previous fractures (38\% versus $21 \%$ ) compared with those who did not die of CVD [72].

In other rheumatic diseases, such as SLE, the information on CV risk in OP is limited. The only studies published used surrogate markers for CVD, such as carotid ultrasound rather than clinical CVD data. Ramsey-Goldman and Manzi [14] reported associations between more carotid plaques or coronary calcification and low BMD in young patients with SLE.

Autoantibodies play a major role in the pathogenesis of RA and other autoimmune diseases. Anti-citrullinated protein antibodies (ACPAs) and rheumatoid factor (RF), which play an essential role in RA, may also be associated with atherogenesis and bone loss. Indeed, both ACPA and RF positivity have been associated with increased $\mathrm{CV}$ risk in RA [73]. Furthermore, ACPA and RF, even independently of inflammation, may synergistically induce bone resorption in RA [74].

In conclusion, inflammatory processes and underlying inflammatory diseases may enhance the development of both atherosclerosis and OP. "Accelerated" or "inflammatory" atherosclerosis and bone loss have been associated with RA and other inflammatory conditions. Therefore, such patients should be routinely screened for CVD and OP.

\section{Possibilities of parallel targeting of cardiovascular disease and osteoporosis}

Although some anti-OP drugs may have favorable $\mathrm{CV}$ effects and inversely some vasoactive agents may have positive effects on the bone, it is more likely that in the case of arthritides and autoimmune diseases, treatment of the underlying disease may also improve CV and OP comorbidities $[4,6,19]$. There have been multiple studies in this field, so here we only briefly summarize general considerations.

Vitamin D has been considered an essential background treatment and preventative compound in OP. Vitamin D deficiency has also been associated with T1DM and CVD [42, 45]. Furthermore, vitamin D exerts numerous immunomodulatory properties and thus may be used in immuno-inflammatory diseases [42, 45].

Calcium supplementation has been implicated in the development of CVD in the general population, but this issue is still under debate. This association was 
suggested by some large studies (for example, NIH-AARP) but conflicted by others (for example, Framingham and the Women's Health Initiative) [72, 75]. Interestingly, according to a recent study, calcium supplementation together with high (but not with low) ESR was associated with increased all-cause and CV mortality of patients with RA [72]. However, it is difficult to evaluate calcium supplementation as a CV risk in RA. It is possible that calcium was mostly prescribed to RA patients with OP who already have a high background risk for CVD.

Among anti-OP agents, bisphosphonates in some studies inhibited atherogenesis and significantly decreased serum low-density lipoprotein (LDL) and increased high-density lipoprotein (HDL) in postmenopausal women [20]. Yet the effect of bisphosphonates on CV risk is not clear, but a reduction in mortality was prescribed for zoledronic acid after a hip fracture [76]. As described above, OPG has been linked to vascular calcification and CVD [36]. Therefore, it is an important question whether RANKL inhibition by denosumab would affect the CV system. Samelson et al. [77] compared more than 2300 denosumab- or placebotreated patients from the FREEDOM (Fracture Reduction Evaluation of Denosumab in Osteoporosis every 6 Months) trial. RANKL inhibition had no effect at all on the progression of arterial calcification or on the incidence of $\mathrm{CV}$ events [77].

On the other hand, statins and nitrates, agents used in vasculoprotection, may also have beneficial effects on the bone $[20,78]$. Weaker evidence suggests that thiazide diuretics and $\beta$-blockers may also exert favorable effects on bone [78]. By contrast, some drugs, such as loop-acting diuretics and warfarin, may aggravate bone loss [78]. There have been very few prospective trials in this respect, so the effects of heart drugs on bone should be confirmed by future studies [78].

With respect to anti-rheumatic and anti-inflammatory drugs used to treat RA and other inflammatory rheumatic diseases, there has been a lot of controversy on the benefits versus risks of low-dose corticosteroid treatment. In general, corticosteroids indeed may be pro-atherogenic [79] and may stimulate bone loss [80]. However, in RA, the anti-inflammatory effects of low-dose corticosteroid treatment may be beneficial for vasculature and bone. The net effect of corticosteroids on CVD and OP may vary in different diseases and patients $[4,5,26,79-82]$. According to a recent OP consensus paper by the ESCEO (European Society for Clinical and Economic Aspects of Osteoporosis and Osteoarthritis) [81] and the recent EULAR CV recommendations [4], short durations and moderate doses of glucocorticoids are recommended. In this case, corticosteroids are generally well tolerated and have a positive benefit/risk ratio. Yet patients on corticosteroid therapy should be regularly assessed for CV and FF risk and, if needed, should be treated $[4,80,81]$.

Among conventional synthetic disease-modifying antirheumatic drugs (csDMARDs), methotrexate (MTX) may exert beneficial effects on bone and on the CV system primarily by controlling systemic inflammation $[4,5,26,82]$. MTX has not only indirect but also direct effects on bone resorption [83] and lipid metabolism [5].

In brief, biologic DMARDs (bDMARD), primarily TNF inhibitors, may also decrease inflammatory atherogenesis $[5,17,19,84]$, may lower the risk of CVD $[4,5$, $19,82,85,86]$, and may halt periarticular and generalized bone loss $[6,26,87,88]$ in inflammatory rheumatic diseases. Moreover, according to a recent study, RA patients receiving TNF inhibitors had even lower risk of MI compared with those treated with csDMARDs [86]. In a recent prospective early RA cohort, the use of biologics was inversely associated with the risk of comorbidity development [25].

Finally, in addition to the EULAR recommendations on CVD management in rheumatic diseases [4] and the recent EULAR/European Federation of National Associations of Orthopaedics and Traumatology (EULAR/ EFORT) recommendations on the management of FF [9], a EULAR initiative published "points to consider" for reporting, screening, and preventing comorbidities in patients with chronic inflammatory rheumatic diseases. This paper focuses on six comorbidities, including CVD and OP. These recommendations also include advice on lifestyle modifications in addition to drug therapy [89].

In conclusion, one can set up a holistic approach to the management of CVD and OP, especially in patients with inflammatory rheumatic diseases. The understanding of common pathogenic factors, as molecular targets, may help us to design novel strategies that combat both $\mathrm{CV}$ disease and bone loss.

\section{Conclusions}

In an aging population, comorbidities such as CVD or OP occur more frequently. These comorbidities develop even more often in patients with inflammatory rheumatic diseases such as RA and SLE. These interactions are plotted in Fig. 1 as a "Bermuda triangle". Although rheumatologists nowadays are successful in treating their patients according to the treat-to-target design (particularly in RA), it is obvious that this single disease framework may not cover all comorbidities; therefore, comorbidities are still suboptimally prevented, screened, and managed. In this review, we discussed how the epidemiology and pathogenesis of $\mathrm{CV}$ events and $\mathrm{OP}$ are strikingly overlapping. Theoretically, smoking, DM, and sedentary lifestyle as conventional risk factors and systemic inflammation are among the modifiable risk factors for both atherosclerosis and bone loss. A holistic approach to treatment may 
involve the use of drugs and lifestyle modifications that may have beneficial effects on bone as well as on vasculature. In RA patients with accelerated inflammatory atherosclerosis and bone loss, the rapid and effective suppression of inflammation by corticosteroids, csDMARDs, and bDMARDs in a treat-to-target manner aiming at clinical remission is necessary to effectively control comorbidities.

One of the limitations of the reviewed dataset is that most of the studies have been conducted in Caucasian women; thus, extrapolation to men and women of different genetic background is not possible. Another issue is that the associations of CVD and OP in patients with RA are highly dependent on the underlying systemic inflammation. Thus, it is not clear whether a similar relationship would be valid in modern times, when RA therapy is aiming at clinical remission. Finally, possibly the most important limitation is that almost no data have been presented on the relationship between cumulative disease activity, functional capacity and/or radiological damage and the risk of $\mathrm{CV}$ events and/or OP in patients with arthritis. For example, in the recent study by $\mathrm{Ni}$ Mhuircheartaigh et al. [70] discussed above, the risk of a CV event is $80 \%$ higher in RA patients after a fracture but is likely to be even higher in RA patients with high disease activity and lower in patients in clinical remission. Thus, there is still a lot of work to do both in research and in daily practice: the conduction of more prospective studies that assess the epidemiological, clinical, and pathophysiological characteristics of CVD and $\mathrm{OP}$ in parallel is urgently needed, but optimal treatment aiming at remission and advocating lifestyle factors in all our patients is also a challenge.

\section{Abbreviations \\ ACPA: Anti-citrullinated protein antibody; bDMARD: Biologic disease- modifying anti-rheumatic drug; BMD: Bone mineral density; BMP: Bone morphogenetic protein; CAC: Coronary artery calcium; CIMT: Carotid intima- media thickness; CKD: Chronic kidney disease; CRP: C-reactive protein: CSDMARD: Conventional synthetic disease-modifying anti-rheumatic drug; CV: Cardiovascular; CVD: Cardiovascular disease; DM: Diabetes mellitus; DMARD: Disease-modifying anti-rheumatic drug; ESR: Erythrocyte sedimentation rate; EULAR: European League Against Rheumatism; FF: Fragility fracture; FGF-23: Fibroblast growth factor 23; HR: Hazard ratio; IL: Interleukin; MI: Myocardial infarction; MTX: Methotrexate; OP: Osteoporosis; OPG: Osteoprotegerin; OPN: Osteopontin; OR: Odds ratio; PTH: Parathyroid hormone; RA: Rheumatoid arthritis; RANKL: Receptor activator of nuclear factor kappa B ligand; RF: Rheumatoid factor; SD: Standard deviation; SLE: Systemic lupus erythematosus; SOST: Sclerostin; SpA: Spondyloarthritis; T1DM: Type 1 diabetes mellitus; TNF: Tumor necrosis factor}

\section{Acknowledgments}

This research was supported by the European Union and the State of Hungary and co-financed by the European Social Fund in the framework of TAMOP4.2.4.A/2-11/1-2012-0001 'National Excellence Program' (ZS) and received support from European Union grants GINOP-2.3.2-15-2016-00015 and GINOP2.3.2-15-2016-00050 (ZS).

Availability of data and materials Not applicable.
Authors' contributions

ZS, HGR, ZP, WFL: all took part in collecting literature data, writing article. All authors read and approved the final manuscript.

Ethics approval and consent to participate

Not applicable.

\section{Consent for publication}

Not applicable.

Competing interests

The authors declare that they have no competing interests.

\section{Publisher's Note}

Springer Nature remains neutral with regard to jurisdictional claims in published maps and institutional affiliations.

\section{Author details}

${ }^{1}$ Division of Rheumatology, Department of Internal Medicine, Faculty of Medicine, University of Debrecen, Nagyerdei street 98, Debrecen 4032, Hungary. ${ }^{2}$ Department of Rheumatology, Northwest Clinics, Wilhelminalaan 12, Alkmaar, JD 1815, The Netherlands. ${ }^{3}$ Amsterdam Rheumatology and Immunology Centre, Location VU University Medical Centre, Amsterdam 1007 MB, Amsterdam 1104 LP, The Netherlands.

Published online: 10 January 2019

\section{References}

1. Radner H, Yoshida K, Smolen JS, Solomon DH. Multimorbidity and rheumatic conditions-enhancing the concept of comorbidity. Nat Rev Rheumatol. 2014;10:252-6.

2. Barnett K, Mercer SW, Norbury M, Watt G, Wyke S, Guthrie B. Epidemiology of multimorbidity and implications for health care, research, and medical education: a cross-sectional study. Lancet. 2012;380:37-43.

3. Prabhakaran D, Anand S, Watkins D, Gaziano T, Wu Y, Mbanya JC, Disease Control Priorities-3 Cardiovascular R, Related Disorders Author G, et al. Cardiovascular, respiratory, and related disorders: key messages from Disease Control Priorities, 3rd edition. Lancet. 2018;391:1224-36.

4. Agca R, Heslinga SC, Rollefstad S, Heslinga M, Mclnnes IB, Peters MJ, et al. EULAR recommendations for cardiovascular disease risk management in patients with rheumatoid arthritis and other forms of inflammatory joint disorders: 2015/2016 update. Ann Rheum Dis. 2017;76:17-28.

5. Szekanecz Z, Kerekes G, Vegh E, Kardos Z, Barath Z, Tamasi L, et al. Autoimmune atherosclerosis in 3D: How it develops, how to diagnose and what to do. Autoimmun Rev. 2016;15:756-69.

6. Szentpetery A, Horvath A, Gulyas K, Petho Z, Bhattoa HP, Szanto S, et al. Effects of targeted therapies on the bone in arthritides. Autoimmun Rev. 2017;16:313-20.

7. Salman-Monte TC, Torrente-Segarra V, Vega-Vidal AL, Corzo P, CastroDominguez F, Ojeda F, et al. Bone mineral density and vitamin D status in systemic lupus erythematosus (SLE): A systematic review. Autoimmun Rev. 2017;16:1155-9.

8. Lems WF, Dijkmans BA. Should we look for osteoporosis in patients with rheumatoid arthritis? Ann Rheum Dis. 1998;57:325-7.

9. Lems WF, Dreinhofer KE, Bischoff-Ferrari H, Blauth M, Czerwinski E, da Silva J, et al. EULAR/EFORT recommendations for management of patients older than 50 years with a fragility fracture and prevention of subsequent fractures. Ann Rheum Dis. 2017:76:802-10.

10. Lems WF, Raterman HG. Critical issues and current challenges in osteoporosis and fracture prevention. An overview of unmet needs. Ther Adv Musculoskelet Dis. 2017;9:299-316.

11. Nurmohamed MT, Heslinga M, Kitas GD. Cardiovascular comorbidity in rheumatic diseases. Nat Rev Rheumatol. 2015;11:693-704.

12. Shoenfeld Y, Gerli R, Doria A, Matsuura E, Cerinic MM, Ronda N, et al. Accelerated atherosclerosis in autoimmune rheumatic diseases. Circulation. 2005; 112:3337-47.

13. Dougados $M$, Soubrier $M$, Antunez A, Balint $P$, Balsa A, Buch $M H$, et al Prevalence of comorbidities in rheumatoid arthritis and evaluation of their monitoring: results of an international, cross-sectional study (COMORA). Ann Rheum Dis. 2014;73:62-8. 
14. Ramsey-Goldman R, Manzi S. Association of osteoporosis and cardiovascular disease in women with systemic lupus erythematosus. Arthritis Rheum. 2001;44:2338-41

15. Symmons DP, Gabriel SE. Epidemiology of CVD in rheumatic disease, with a focus on RA and SLE. Nat Rev Rheumatol. 2011;7:399-408.

16. Takayanagi H. New developments in osteoimmunology. Nat Rev Rheumatol. 2012;8:684-9.

17. Szekanecz Z, Kerekes G, Kardos Z, Baráth Z, Tamási L. Mechanisms of inflammatory atherosclerosis in rheumatoid arthritis. Curr Immunol Rev. 2016;12:35-46.

18. Ross R. Atherosclerosis--an inflammatory disease. N Engl J Med. 1999;340:115-26.

19. Szekanecz Z, Kerekes G, Soltesz P. Vascular effects of biologic agents in RA and spondyloarthropathies. Nat Rev Rheumatol. 2009;5:677-84.

20. McFarlane SI, Muniyappa R, Shin JJ, Bahtiyar G, Sowers JR. Osteoporosis and cardiovascular disease: brittle bones and boned arteries, is there a link? Endocrine. 2004;23:1-10.

21. Laroche M, Pecourneau V, Blain H, Breuil V, Chapurlat R, Cortet B, et al. Osteoporosis and ischemic cardiovascular disease. Joint Bone Spine. 2017; 84:427-32.

22. Laakso M, Kuusisto J. Epidemiological evidence for the association of hyperglycaemia and atherosclerotic vascular disease in non-insulindependent diabetes mellitus. Ann Med. 1996;28:415-8.

23. Napoli N, Chandran M, Pierroz DD, Abrahamsen B, Schwartz AV, Ferrari SL, et al. Mechanisms of diabetes mellitus-induced bone fragility. Nat Rev Endocrinol. 2017;13:208-19.

24. Lampropoulos CE, Papaioannou I, D'Cruz DP. Osteoporosis--a risk factor for cardiovascular disease? Nat Rev Rheumatol. 2012;8:587-98.

25. Innala L, Sjoberg C, Moller B, Ljung L, Smedby T, Sodergren A, et al. Comorbidity in patients with early rheumatoid arthritis - inflammation matters. Arthritis Res Ther. 2016:18:33.

26. Hoes JN, Bultink IE, Lems WF. Management of osteoporosis in rheumatoid arthritis patients. Expert Opin Pharmacother. 2015;16:559-71.

27. Sambrook P, Cooper C. Osteoporosis. Lancet. 2006;367:2010-8.

28. Galliera E, Locati M, Mantovani A, Corsi MM. Chemokines and bone remodeling. Int J Immunopathol Pharmacol. 2008;21:485-91.

29. Ridker PM, Rifai N, Pfeffer M, Sacks F, Lepage S, Braunwald E. Elevation of tumor necrosis factor-alpha and increased risk of recurrent coronary events after myocardial infarction. Circulation. 2000;101:2149-53.

30. Barbour KE, Boudreau R, Danielson ME, Youk AO, Wactawski-Wende J, Greep NC, et al. Inflammatory markers and the risk of hip fracture: the Women's Health Initiative. J Bone Miner Res. 2012;27:1167-76.

31. Gomez R, Conde J, Scotece M, Gomez-Reino JJ, Lago F, Gualillo O. What's new in our understanding of the role of adipokines in rheumatic diseases? Nat Rev Rheumatol. 2011;7:528-36.

32. Kerekes G, Nurmohamed MT, Gonzalez-Gay MA, Seres I, Paragh G, Kardos Z, et al. Rheumatoid arthritis and metabolic syndrome. Nat Rev Rheumatol. 2014;10:691-6.

33. Neumann E, Junker S, Schett G, Frommer K, Muller-Ladner U. Adipokines in bone disease. Nat Rev Rheumatol. 2016;12:296-302.

34. Varri M, Niskanen L, Tuomainen T, Honkanen R, Kroger H, Tuppurainen MT. Association of adipokines and estradiol with bone and carotid calcifications in postmenopausal women. Climacteric. 2016;19:204-11.

35. Shimizu H, Nakagami H, Osako MK, Hanayama R, Kunugiza Y, Kizawa T, et al. Angiotensin II accelerates osteoporosis by activating osteoclasts. FASEB J. 2008;22:2465-75

36. Kiechl S, Werner P, Knoflach M, Furtner M, Willeit J, Schett G. The osteoprotegerin/RANK/RANKL system: a bone key to vascular disease. Expert Rev Cardiovasc Ther. 2006:4:801-11.

37. Schett G, Gravallese E. Bone erosion in rheumatoid arthritis: mechanisms, diagnosis and treatment. Nat Rev Rheumatol. 2012;8:656-64.

38. Venuraju SM, Yerramasu A, Corder R, Lahiri A. Osteoprotegerin as a predictor of coronary artery disease and cardiovascular mortality and morbidity. J Am Coll Cardiol. 2010;55:2049-61.

39. Wehmeyer C, Frank S, Beckmann D, Bottcher M, Cromme C, Konig U, et al. Sclerostin inhibition promotes TNF-dependent inflammatory joint destruction. Sci Transl Med. 2016;8:330ra335

40. Gaudio A, Fiore V, Rapisarda R, Sidoti MH, Xourafa A, Catalano A, et al. Sclerostin is a possible candidate marker of arterial stiffness: Results from a cohort study in Catania. Mol Med Rep. 2017;15:3420-4.

41. Morrell NW, Bloch DB, ten Dijke P, Goumans MJ, Hata A, Smith J, et al. Targeting BMP signalling in cardiovascular disease and anaemia. Nat Rev Cardiol. 2016;13:106-20.
42. Holick MF. Sunlight and vitamin D for bone health and prevention of autoimmune diseases, cancers, and cardiovascular disease. Am J Clin Nutr. 2004;80(6 Suppl):1678S-88S.

43. Carvalho LS, Sposito AC. Vitamin D for the prevention of cardiovascular disease: Are we ready for that? Atherosclerosis. 2015;241:729-40.

44. Muscogiuri G, Annweiler C, Duval G, Karras S, Tirabassi G, Salvio G, et al. Vitamin D and cardiovascular disease: From atherosclerosis to myocardial infarction and stroke. Int J Cardiol. 2017;230:577-84.

45. Arnson $Y$, Amital H, Shoenfeld Y. Vitamin D and autoimmunity: new aetiological and therapeutic considerations. Ann Rheum Dis. 2007;66: 1137-42.

46. Brandenburg VM, Kleber ME, Vervloet MG, Tomaschitz A, Pilz S, Stojakovic T, et al. Fibroblast growth factor 23 (FGF23) and mortality: the Ludwigshafen Risk and Cardiovascular Health Study. Atherosclerosis. 2014;237:53-9.

47. Mirza MA, Karlsson MK, Mellstrom D, Orwoll E, Ohlsson C, Ljunggren O, et al. Serum fibroblast growth factor-23 (FGF-23) and fracture risk in elderly men. J Bone Miner Res. 2011;26:857-64.

48. Scatena M, Liaw L, Giachelli CM. Osteopontin: a multifunctional molecule regulating chronic inflammation and vascular disease. Arterioscler Thromb Vasc Biol. 2007;27:2302-9.

49. Lutgens E, Lutgens SP, Faber BC, Heeneman S, Gijbels MM, de Winther MP, et al. Disruption of the cathepsin $\mathrm{K}$ gene reduces atherosclerosis progression and induces plaque fibrosis but accelerates macrophage foam cell formation. Circulation. 2006;113:98-107.

50. Massy ZA, Drueke TB. Vascular calcification. Curr Opin Nephrol Hypertens. 2013;22:405-12.

51. Byon $\mathrm{CH}$, Chen Y. Molecular Mechanisms of Vascular Calcification in Chronic Kidney Disease: The Link between Bone and the Vasculature. Curr Osteoporos Rep. 2015;13:206-15.

52. Zhang $Y$, Feng B. Systematic review and meta-analysis for the association of bone mineral density and osteoporosis/osteopenia with vascular calcification in women. Int J Rheum Dis. 2017:20:154-60.

53. Aleksova J, Kurniawan S, Vucak-Dzumhur M, Kerr P, Ebeling PR, Milat F, et al. Aortic vascular calcification is inversely associated with the trabecular bone score in patients receiving dialysis. Bone. 2018;113:118-23.

54. Barengolts El, Berman M, Kukreja SC, Kouznetsova T, Lin C, Chomka EV. Osteoporosis and coronary atherosclerosis in asymptomatic postmenopausal women. Calcif Tissue Int. 1998:62:209-13.

55. Campos-Obando N, Kavousi M, Roeters van Lennep JE, Rivadeneira F, Hofman A, Uitterlinden AG, et al. Bone health and coronary artery calcification: The Rotterdam Study. Atherosclerosis. 2015;241:278-83.

56. Shaffer JR, Kammerer CM, Rainwater DL, O'Leary DH, Bruder JM, Bauer RL, et al. Decreased bone mineral density is correlated with increased subclinical atherosclerosis in older, but not younger, Mexican American women and men: the San Antonio Family Osteoporosis Study. Calcif Tissue Int. 2007;81: 430-41.

57. Delgado-Frias E, Lopez-Mejias R, Genre F, Ubilla B, Gomez RodriguezBethencourt MA, Gonzalez-Diaz A, et al. Relationship between endothelial dysfunction and osteoprotegerin, vitamin $\mathrm{D}$, and bone mineral density in patients with rheumatoid arthritis. Clin Exp Rheumatol. 2015;33:241-9.

58. Ye C, Xu M, Wang S, Jiang S, Chen X, Zhou X, et al. Decreased Bone Mineral Density Is an Independent Predictor for the Development of Atherosclerosis: A Systematic Review and Meta-Analysis. PLoS One. 2016;11: e0154740

59. Veronese N, Stubbs B, Crepaldi G, Solmi M, Cooper C, Harvey NC, et al. Relationship Between Low Bone Mineral Density and Fractures With Incident Cardiovascular Disease: A Systematic Review and Meta-Analysis. J Bone Miner Res. 2017;32:1126-35.

60. Jin ES, Jeong JH, Lee B, Im SB. Association factor analysis between osteoporosis with cerebral artery disease: The STROBE study. Medicine (Baltimore). 2017;96:e6164

61. Varosy PD, Shlipak MG, Vittinghoff E, Black DM, Herrington D, Hulley SB, Heart, Estrogen/Progestin Replacement Study I, et al. Fracture and the risk of coronary events in women with heart disease. Am J Med. 2003; 115:196-202.

62. Lems WF. Is Fragility Fracture a Strong Risk Factor for a Cardiovascular Event in Rheumatoid Arthritis? The Challenge of Dealing with Multiple Comorbidities. J Rheumatol. 2017:44:545-6.

63. Qu X, Huang X, Jin F, Wang H, Hao Y, Tang T, et al. Bone mineral density and all-cause, cardiovascular and stroke mortality: a meta-analysis of prospective cohort studies. Int J Cardiol. 2013;166:385-93. 
64. Domiciano DS, Machado LG, Lopes JB, Figueiredo CP, Caparbo VF, Oliveira RM, et al. Bone Mineral Density and Parathyroid Hormone as Independent Risk Factors for Mortality in Community-Dwelling Older Adults: A Population-Based Prospective Cohort Study in Brazil. The Sao Paulo Ageing \& Health (SPAH) Study. J Bone Miner Res. 2016;31:1146-57.

65. den Uyl D, Nurmohamed MT, van Tuyl LH, Raterman HG, Lems WF. (Sub)clinical cardiovascular disease is associated with increased bone loss and fracture risk; a systematic review of the association between cardiovascular disease and osteoporosis. Arthritis Res Ther. 2011;13:R5.

66. Sennerby U, Melhus H, Gedeborg R, Byberg L, Garmo H, Ahlbom A, et al Cardiovascular diseases and risk of hip fracture. JAMA. 2009;302:1666-73.

67. Gerber Y, Melton $L J$ 3rd, Weston SA, Roger VL. Association between myocardial infarction and fractures: an emerging phenomenon. Circulation. 2011;124:297-303.

68. Pouwels S, Lalmohamed A, Leufkens B, de Boer A, Cooper C, van Staa T, et al. Risk of hip/femur fracture after stroke: a population-based case-control study. Stroke 2009:40:3281-3285.

69. van Staa TP, Geusens P, Bijlsma JW, Leufkens HG, Cooper C. Clinical assessment of the long-term risk of fracture in patients with rheumatoid arthritis. Arthritis Rheum. 2006;54:3104-12.

70. Ni Mhuircheartaigh O, Crowson CS, Gabriel SE, Roger VL, Melton LJ 3rd, Amin S. Fragility Fractures Are Associated with an Increased Risk for Cardiovascular Events in Women and Men with Rheumatoid Arthritis: A Population-based Study. J Rheumatol. 2017;44:558-64.

71. Popescu C, Bojinca V, Opris D, lonescu R. Whole body bone tissue and cardiovascular risk in rheumatoid arthritis. J Osteoporos. 2014;2014:465987.

72. Provan SA, Olsen IC, Austad C, Haugeberg G, Kvien TK, Uhlig T. Calcium supplementation and inflammation increase mortality in rheumatoid arthritis: A 15-year cohort study in 609 patients from the Oslo Rheumatoid Arthritis Register. Semin Arthritis Rheum. 2017;46:411-7.

73. Cambridge G, Acharya J, Cooper JA, Edwards JC, Humphries SE. Antibodies to citrullinated peptides and risk of coronary heart disease. Atherosclerosis. 2013;228:243-6.

74. Hecht C, Englbrecht M, Rech J, Schmidt S, Araujo E, Engelke K, et al. Additive effect of anti-citrullinated protein antibodies and rheumatoid factor on bone erosions in patients with RA. Ann Rheum Dis. 2015;74:2151-6.

75. Rautiainen S, Wang L, Manson JE, Sesso HD. The role of calcium in the prevention of cardiovascular disease--a review of observational studies and randomized clinical trials. Curr Atheroscler Rep. 2013;15:362.

76. Lyles KW, Colon-Emeric CS, Magaziner JS, Adachi JD, Pieper CF, Mautalen C, et al. Zoledronic acid and clinical fractures and mortality after hip fracture. $\mathrm{N}$ Engl J Med. 2007;357:1799-809.

77. Samelson EJ, Miller PD, Christiansen C, Daizadeh NS, Grazette L, Anthony MS, et al. RANKL inhibition with denosumab does not influence 3-year progression of aortic calcification or incidence of adverse cardiovascular events in postmenopausal women with osteoporosis and high cardiovascular risk. J Bone Miner Res. 2014;29:450-7.

78. Walsh JS, Newman C, Eastell R. Heart drugs that affect bone. Trends Endocrinol Metab. 2012;23:163-8.

79. Nurmohamed MT, van Halm VP, Dijkmans BA. Cardiovascular risk profile of antirheumatic agents in patients with osteoarthritis and rheumatoid arthritis. Drugs. 2002;62:1599-609.

80. Buckley L, Guyatt G, Fink HA, Cannon M, Grossman J, Hansen KE, et al. 2017 American College of Rheumatology Guideline for the Prevention and Treatment of Glucocorticoid-Induced Osteoporosis. Arthritis Rheumatol. 2017;69:1521-37.

81. Cooper C, Bardin T, Brandi ML, Cacoub P, Caminis J, Civitelli R, et al. Balancing benefits and risks of glucocorticoids in rheumatic diseases and other inflammatory joint disorders: new insights from emerging data. An expert consensus paper from the European Society for Clinical and Economic Aspects of Osteoporosis and Osteoarthritis (ESCEO). Aging Clin Exp Res. 2016;28:1-16.

82. Roubille C, Richer V, Starnino T, McCourt C, McFarlane A, Fleming P, et al. The effects of tumour necrosis factor inhibitors, methotrexate, non-steroidal anti-inflammatory drugs and corticosteroids on cardiovascular events in rheumatoid arthritis, psoriasis and psoriatic arthritis: a systematic review and meta-analysis. Ann Rheum Dis. 2015;74:480-9.

83. Torikai E, Kageyama Y, Takahashi M, Nagano A. The effect of methotrexate on bone metabolism markers in patients with rheumatoid arthritis. Mod Rheumatol. 2006:16:350-4.
84. Szekanecz Z, Szanto S, Szabo Z, Vancsa A, Szamosi S, Bodnar N, et al. Biologics - beyond the joints. Autoimmun Rev. 2010;9:820-4.

85. Greenberg JD, Furer V, Farkouh ME. Cardiovascular safety of biologic therapies for the treatment of RA. Nat Rev Rheumatol. 2011;8:13-21.

86. Low AS, Symmons DP, Lunt M, Mercer LK, Gale CP, Watson KD, British Society for Rheumatology Biologics Register for Rheumatoid A, the BCCC, et al. Relationship between exposure to tumour necrosis factor inhibitor therapy and incidence and severity of myocardial infarction in patients with rheumatoid arthritis. Ann Rheum Dis. 2016;76:654-60.

87. Vis M, Havaardsholm EA, Haugeberg G, Uhlig T, Voskuyl AE, van de Stadt RJ, et al. Evaluation of bone mineral density, bone metabolism, osteoprotegerin and receptor activator of the NFkappaB ligand serum levels during treatment with infliximab in patients with rheumatoid arthritis. Ann Rheum Dis. 2006;65:1495-9.

88. Krieckaert CL, Nurmohamed MT, Wolbink G, Lems WF. Changes in bone mineral density during long-term treatment with adalimumab in patients with rheumatoid arthritis: a cohort study. Rheumatology (Oxford). 2013;52: 547-53.

89. Baillet A, Gossec L, Carmona L, Wit M, van Eijk-Hustings $Y$, Bertheussen $H$, et al. Points to consider for reporting, screening for and preventing selected comorbidities in chronic inflammatory rheumatic diseases in daily practice: a EULAR initiative. Ann Rheum Dis. 2016:75:965-73.

\section{Ready to submit your research? Choose BMC and benefit from:}

- fast, convenient online submission

- thorough peer review by experienced researchers in your field

- rapid publication on acceptance

- support for research data, including large and complex data types

- gold Open Access which fosters wider collaboration and increased citations

- maximum visibility for your research: over $100 \mathrm{M}$ website views per year

At BMC, research is always in progress.

Learn more biomedcentral.com/submissions 